\title{
Preliminary study to the temperatures of the thermocouple probes affected by the environment of heated walls
}

\author{
Richard Matas ${ }^{1, a}$, Jan Sedláček ${ }^{1}$ and Václav Čibera ${ }^{1}$ \\ ${ }^{1}$ New Technologies - Research Centre, University of West Bohemia, Univerzitni 8, 30614 Pilsen, Czech Republic
}

\begin{abstract}
Measuring of the total temperatures of the flowing medium is one of the pivotal points in the development when a new compressor stages are tested. Measurements conducted on the test compressor indicated that in the specific cases the measured temperature can be affected by the temperature of the walls which hold thermocouple probe. By implication of this observed fact, the preliminary study about this phenomenon was investigated. It was considered the influence of the heat conduction in the body of the probe and in the thermocouples. The main aim of the study was to examine the properties and the behaviour of the thermocouple probes in various working regimes. The preliminary study was focused on the development of the tools that can be used for complex solution of the problem by using of measured data. The results of this study might help to correct measured data in the case of skewed measurements affected by surroundings of the thermocouple probes.
\end{abstract}

\section{Introduction}

The measurements of the total temperatures are one of the crucial steps within verifying of the parameters of the new compressor stages [1]. The accurate values of the measured temperatures are necessary for the evaluation and determining the efficiency and other properties. Further, the correct measured temperatures are important for design documents as well as for comparison with results of CFD simulations [2].

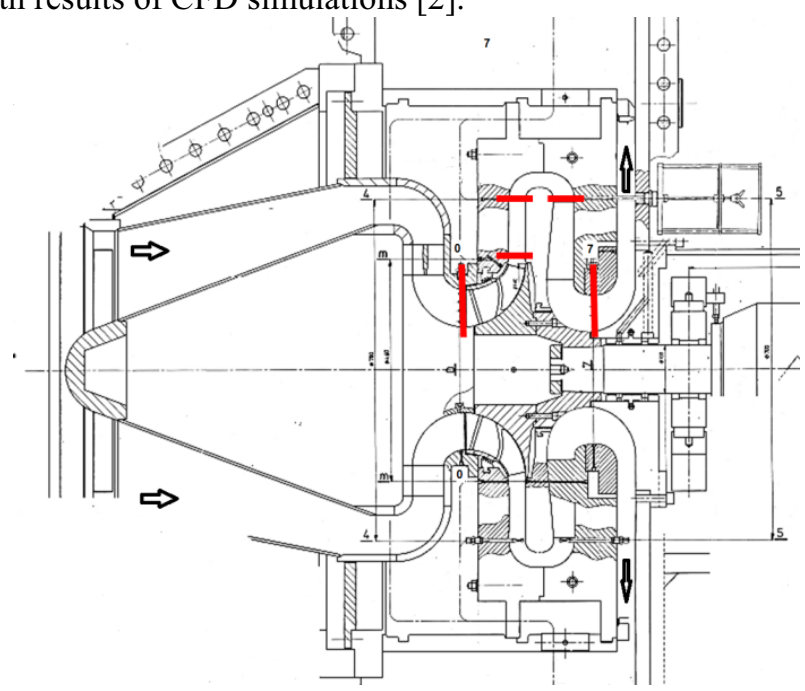

Figure 1. The arrangement of the examined planes in the tested compressor stage.

The probes in different variations have been used to measure total temperatures and total pressure for the testing of the radial compressors. The first type of the probe is a multiple sensors probe containing more than one sensor arranged on the same shank. This kind of probe is used at the inlet and outlet planes of compressor. The second type of probe is the probe containing only a single pressure sensor or a single temperature sensor. This kind of probe is used in inner planes of compressor. The figure 1 shows the arrangement of probes in examined compressor.

The probes are designed and delivered by specialized company to conform demanded high quality. Individual probes are produced to satisfy specific flow cross-section of a compressor stage.

To accomplish complete measurements of temperatures, the temperatures of the inlet and outlet air at orifices have been measured. The temperature of the oil was measured as well by the use of resistance thermometers.

\section{Measuring of the temperatures in a single compressor stage}

The figure 2 shows the real design and details of the used thermocouples of considered probes, which are installed into measuring planes inside compressor. The signals received from these probes are processed and transmitted into control computer. After the correction regarding to the flow rate of air, the signals are used to evaluate the parameters of the compressor stage.

During the measurements it has been assumed that the temperature of the air at the inlet and the outlet is approximately constant across the cross-section of channel. The temperatures across the inner measuring planes might be unequal. And then on the end of the

\footnotetext{
a Corresponding author: mata@ntc.zcu.cz
} 
channel the temperatures across the channel cross-section should be partially equalized. But the measured results for the various conditions seem to indicate that for the various temperatures of the inlet atmospheric air and for the differently warmed parts of the compressor the measured temperature at the inlet cross-section is inhomogeneous. During the observation of this phenomenon, it was confirmed that the temperature measured by the multiple probe was always higher for the sensors closer to the wall of compressor. Contrary, the temperature decreased in the direction to the end of the probe. This effect was amplified when the colder air was sucked in in the course of the measurements during the winter months. In this case the average difference of the temperature at the sensors arranged along the same probe was sometimes more than $1^{\circ} \mathrm{C}$ at the inlet plane. This value can already negatively affect the measured efficiency of the investigated compressor stage. On the other hand, during the summer months, when the warmer air was sucked in, this phenomenon was almost negligible.

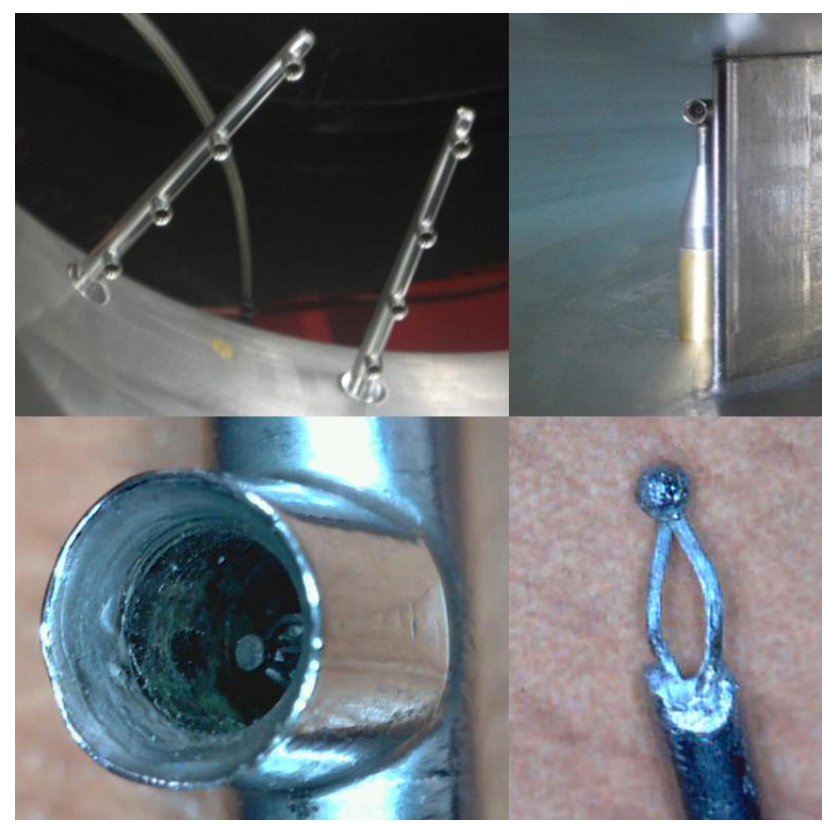

Figure 2. Examples of the probes containing a multiple number of thermocouples and example of the probes with a single thermocouple.

\section{Model of the probe containing three thermocouples}

\subsection{Measured values}

As the reference state served the case with the measurements by using multiple sensors probe containing three thermocouples - the similar with the four thermocouples can be seen in figure 2 up and left. From the recorded measured points, two cases were chosen. These two cases were characterized by the different values for the temperature as well as for the flow rate and so for average inlet velocities 23 and $105 \mathrm{~m} / \mathrm{s}$. The point values of the temperature were the inlet air at the inlet aperture $T_{a}$, the value of the temperature of the wall $T_{w}$. Further, the velocity of the air flow was used as boundary conditions. The average values of the temperatures achieved by the probes on the particular radial positions are used for comparison with the results of simulations.

\subsection{Model of the thermal network}

The first used method for the simulation was the method of thermal network. In this approach, the thermal network is solved as analogy of an electrical circuit. The examined subject is substituted by the elements of electrical circuit. The part of the system is then replaced by elements, usually by $\pi$ elements. The parameters of these elements conform to the physical properties of investigated objects and their relationships to the surroundings environment. This method is often used for design of electrical machines, electronical devices as well as in various other research areas. The advantage of this method is that it provides quick analysis of objects warming in steadystates as well as in transient states.

By use of this method, the multiple sensors probe was divided into 13 sections along its longest directions. Single thermocouples and deflectors we considered. The parameters as thermal resistances of individual sections were computed as substitute values taking into account material properties of appropriate parts and their proportional representation. The coefficient of the heat transfer among the fluid and solid was considered as value depending on thermal gradient among probes and air. Further this coefficient was considered reliant on the velocity of air flow. The computation of the heat transfer coefficient was conducted according to the method introduced in [3].

The computation of thermal network was realized within MATLAB and Simulink environment. Namely, the tool PLECS designed for the analysis of the electrical circuits was used. The solution was computed for different thermal proportions as the temperatures of the walls, thermal gradient according to temperature of flowing air and further for the various velocities of flow.

The results of the simulations are the distribution of the temperature along the probe body, heat flux through the probe, temperatures at the end of the probe and the deviations of the expected values. The warming along the probe for case 1 is showed in the figure 3 .

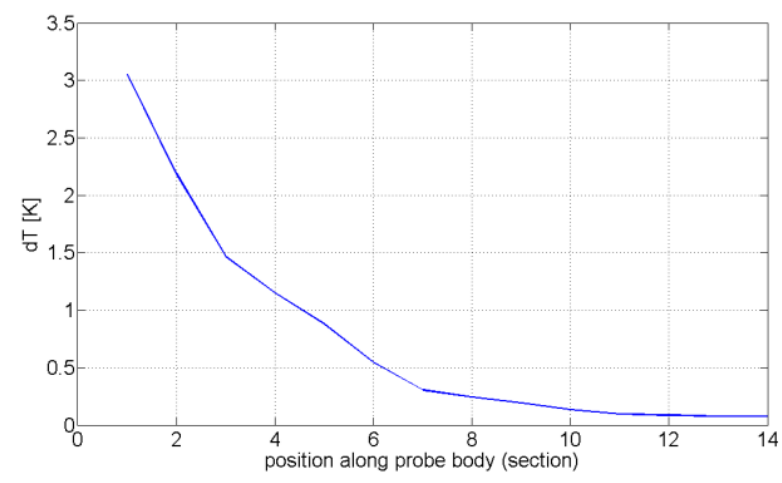

Figure 3. The warming of the probe along its body. The result was achieved by the method of thermal network. 


\subsection{CFD model of the probes with multiple}

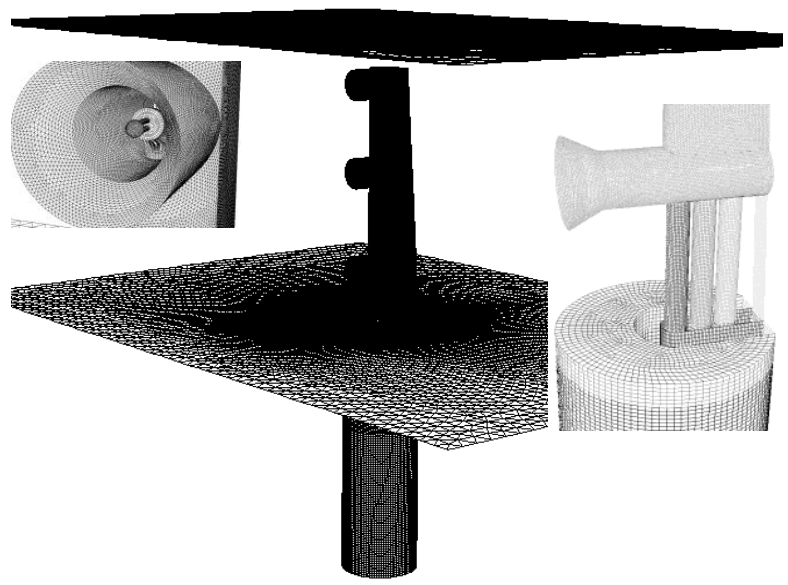

numbers of thermocouples

Figure 4. Surface mesh of the probe model containing three thermocouples and the details of the mesh.

The model for the CFD simulations was assembled for the more detailed investigation of the heat and flow proportions in the probes and in its surroundings. This model contains approximately 4.7 million of the mesh cells. The mesh can be seen in the figure 4 . The model was prepared for the parameters of the real thermocouple probe. The model includes all the geometric properties including the geometry of the inner part of probe and material properties.

The numerical simulation was conducted within the software ANSYS FLUENT 15. The flow of the air was considered as turbulent, rng k- $\varepsilon$ model was used for first simulation. The flowing medium was set as incompressible. The solved case was computed as a steady state. Further, radiation was for the first preliminary results neglected.

The result of the temperature distribution for case 1 is in figure 5. As can be seen from this picture, the warming due to higher temperature of the walls is considerable.
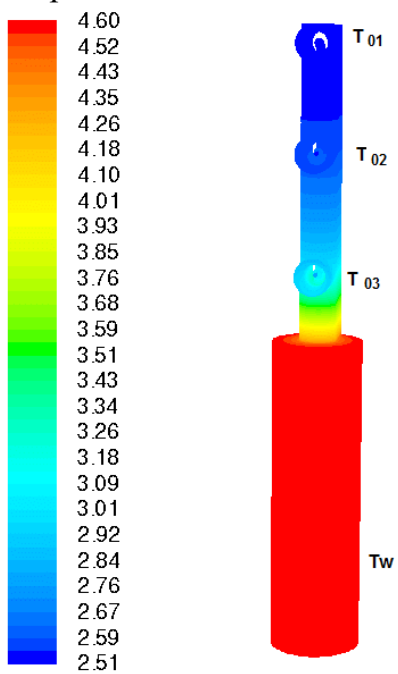

Figure 5. Distribution of the temperature along the probe body.
The figure 6 depicts the cross-section through the probe with surrounding velocity fields. The picture illustratively shows the inhomogeneous velocity fields in the surroundings of the probe body and especially near the thermocouple sensors. The considerable decrease of the flow rate through the deflectors of thermocouple in the direction to the wall of channel can be observed in results of simulation. This might have other negative effects on the measurement of temperature.

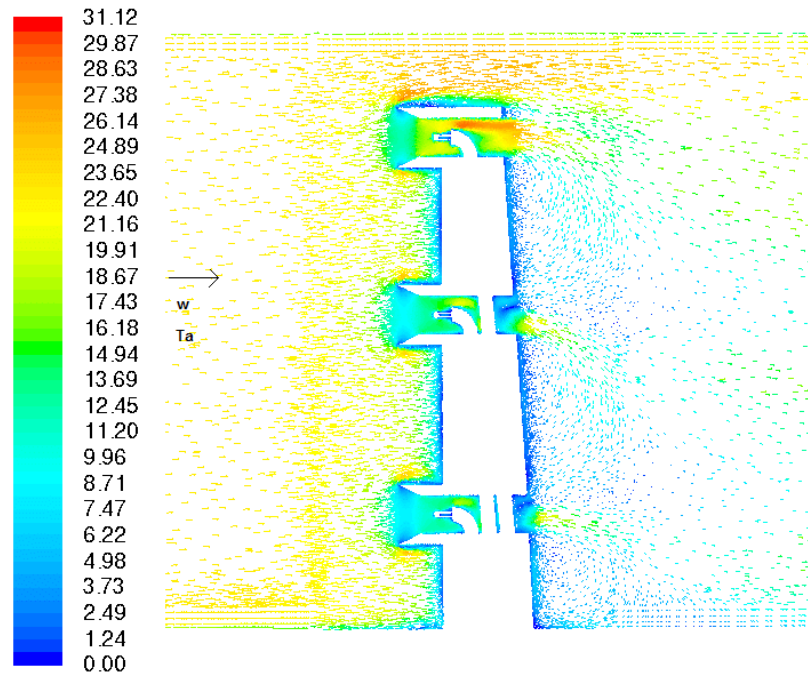

Figure 6. Distribution of the velocity fields near the crosssection of probe - case 1

The preliminary results are summarized in table 1 . The table contains the measured data as well as results of simulation for the chosen points $T_{a}, T_{w}$ and the differences of the temperatures related to the end of probe, where the thermocouple is surrounded with the highest flow rates.

Table 1. Summary of the results achieved by the described methods for both solved cases.

\begin{tabular}{|c|c|c|c|c|c|c|}
\hline & \multicolumn{2}{|c|}{ Measured data } & \multicolumn{2}{|c|}{$\begin{array}{c}\text { Thermal } \\
\text { network } \\
\text { results }\end{array}$} & \multicolumn{2}{c|}{$\begin{array}{c}\text { CFD } \\
\text { results }\end{array}$} \\
\hline $\begin{array}{c}\mathrm{W} \\
{[\mathrm{m} / \mathrm{s}]}\end{array}$ & 23 & 105 & 23 & 105 & 23 & 105 \\
\hline $\begin{array}{c}\mathrm{T}_{\mathrm{a}} \\
{\left[{ }^{\circ} \mathrm{C}\right]}\end{array}$ & 3.05 & 5.6 & 3.05 & 5.6 & 3.05 & 5.6 \\
\hline $\begin{array}{c}\mathrm{T}_{\mathrm{w}} \\
{\left[{ }^{\circ} \mathrm{C}\right]}\end{array}$ & 4.6 & 22.6 & 4.6 & 22.6 & 4.6 & 22.6 \\
\hline $\begin{array}{c}\mathrm{T}_{01} \\
{\left[{ }^{\circ} \mathrm{C}\right]}\end{array}$ & 3.20 & 6.15 & 3.09 & 5.61 & 3.05 & 5.60 \\
\hline $\begin{array}{c}\Delta \mathrm{T}_{12} \\
{\left[{ }^{\circ} \mathrm{C}\right]}\end{array}$ & 0.02 & 0.33 & 0.08 & 0.08 & 0.03 & 0.07 \\
\hline $\begin{array}{c}\mathrm{T}_{02} \\
{\left[{ }^{\circ} \mathrm{C}\right]}\end{array}$ & 3.22 & 6.48 & 3.17 & 5.69 & 3.08 & 5.65 \\
\hline $\begin{array}{c}\Delta \mathrm{T}_{13} \\
{\left[{ }^{\circ} \mathrm{C}\right]}\end{array}$ & 0.22 & 1.98 & 0.54 & 1.07 & 0.20 & 0.68 \\
\hline $\begin{array}{c}\mathrm{T}_{03} \\
{\left[{ }^{\circ} \mathrm{C}\right]}\end{array}$ & 3.42 & 8.13 & 3.63 & 6.67 & 3.25 & 6.28 \\
\hline
\end{tabular}


Besides already mentioned, the next conducted study was the examination of the effect of the used turbulence model. The goal was the determination of the better approach to the comparison of experimental values, because the differences for the case 2 are relative high. The results for case 2 are summarized in the table 2 .

Table 2. Effect of the turbulence model on the results.

\begin{tabular}{|c|c|c|c|}
\hline & rng k- $\varepsilon$ & SST k $-\omega$ & RSM \\
\hline $\begin{array}{c}\mathrm{W} \\
{[\mathrm{m} / \mathrm{s}]}\end{array}$ & \multicolumn{3}{|c|}{105} \\
\hline $\begin{array}{c}\mathrm{T}_{\mathrm{a}} \\
{\left[{ }^{\circ} \mathrm{C}\right]}\end{array}$ & \multicolumn{3}{|c|}{5.6} \\
\hline $\begin{array}{c}\mathrm{T}_{\mathrm{w}} \\
{\left[{ }^{\circ} \mathrm{C}\right]}\end{array}$ & \multicolumn{3}{|c|}{22.6} \\
\hline $\begin{array}{c}\mathrm{T}_{01} \\
{\left[{ }^{\circ} \mathrm{C}\right]}\end{array}$ & 5.60 & 5.60 & 5.61 \\
\hline $\begin{array}{l}\Delta \mathrm{T}_{12} \\
{\left[{ }^{\circ} \mathrm{C}\right]}\end{array}$ & 0.05 & 0.07 & 0.07 \\
\hline $\begin{array}{c}\mathrm{T}_{02} \\
{\left[{ }^{\circ} \mathrm{C}\right]}\end{array}$ & 5.65 & 5.67 & 5.68 \\
\hline $\begin{array}{l}\Delta \mathrm{T}_{13} \\
{\left[{ }^{\circ} \mathrm{C}\right]}\end{array}$ & 0.68 & 0.86 & 0.9 \\
\hline $\begin{array}{c}\mathrm{T}_{03} \\
{\left[{ }^{\circ} \mathrm{C}\right]}\end{array}$ & 6.28 & 6.46 & 6.51 \\
\hline
\end{tabular}

\section{Model of the single thermocouple probe for sensitive analysis}

Since the results achieved at the multiple sensors probe were interesting, it was decided to proceed to the more complex solution including the modelling and simulation of simple probes containing single thermocouples. Other models of probes as depicted in figure 2 were prepared for simulations. The models were prepared for the cases with and without deflectors.

One of the easier cases of the probe with single thermocouple without deflector was simulated within CFD software OpenFOAM 2.4. An example of the simulation results is depicted in figure 7 . The figure shows flow around the thermocouple with heat transfer between solid and fluid, where the fluid warms probe.

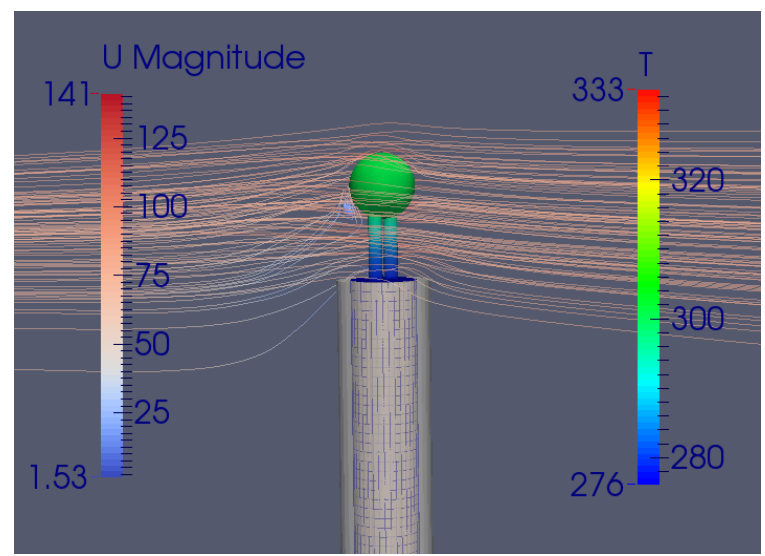

Figure 7. Model of the probe consisting of a single thermocouple. The presented model was prepared in OpenFOAM.

The simulation in OpenFOAM includes radiation. As a radiation model was used view factor model.

\section{Conclusions}

During the measurements of the compressor's stages, the unrealistic and inhomogeneous temperatures were measured in the specific cases. Skewed results were obtained namely at inlet plane of the compressor.

To accomplish the measurements, two different kinds of simulations were conducted. The achieved results of the simulations are in concordance with observed data. That is that the temperatures of the walls affect measurements of total temperatures in the special cases.

The results of the used models unambiguously indicate that there is an impact on the measured total temperatures caused by temperatures of the nearest walls. But it must be admitted that the values achieved by simulation does not correspond with the measured data exactly. On account of this fact, the models still need to be further developed and tuned up to describe the reality more precisely.

Therefore, the simulation models were prepared with the aim for more detailed sensitive analysis of phenomenon. The models include radiation with the aim to examine the impact of the probe surroundings on the particular thermocouple probes.

In the nearest future, the experiments of controlled measurements in the presence of various surrounding conditions are going to be performed. The simulations models are further being developed more sophistically to identify all effects that could have skewed measurements of temperatures of fluid medium through the compressor stages.

\section{Acknowledgement}

These results were achieved in the framework of the FRTI1/126 project of the programme "TIP", Ministry of Industry and Trade of the Czech Republic programme and in specific research by the internal grant at University of West Bohemia: SGS-2014-070.

\section{References}

1. P. C. Hanlon, Compressor handbook McGrawHill, USA (2001)

2. T. Syka, R. Matas, J. Kňourek Hub and shroud fillets influence on the radial compressor stage efficiency, EPJ Web of Conferences, 92 (2015)

3. VDI Heat Atlas $2^{\text {nd }}$ edition, Springer-Verlag Berlin Heidelberg (2010)

4. www.ansys.com

5. www.openfoam.com 